\title{
Navy Telemedicine Initiative
}

\author{
CDR Michael Greenauer, MSC, USN \\ Navy Liaison Officer to U.S. Army Medical and Materiel Command for Telemedicine \\ Department of Navy
}

\section{COMMANDER GREENAUER:}

Thank you Captain Tibbits, and good morning. General, I appreciate the opportunity to discuss this morning the Navy Telemedicine Initiative, an initiative that we have some three years invested in with the Navy Marine Corps Team. When using the term telemedicine we are reminded of the fable of the blind man and the elephant --depending on which piece you have your hands on, each has a different feel. So it is important that we identify what terms we are speaking of when we talk about the Navy Telemedicine Initiative. We are using that as an umbrella term to address the areas that we see on the screen, as well as new and emerging technologies which seem applicable. All of which combines the transportation of digital imaging and clinical data from one point to another or the storage of that data within a facility.

We are using video teleconferencing for teleconsultation. Currently we have telemedicine up and running at over 12 different sites around the world at different components. Video teleconferencing is real-time consultation. We have found that there is an opportunity to conserve clinicians' time because all consults do not have to be real-time. We want to examine the store and forward capabilities in teleconsultation, so we are looking at both real-time and store and forward.

Teletraining is very important to us. We know that by implementing telemedicine, we are also going to have the backbone or infrastructure in place which will allow for clinical training, CME and GME training for our staff at all levels. That is going to result in significant savings for the Navy in travel time and costs for training. We project that possibly more than $30 \%$ of our travel for training will be reduced by having a telepresence for teletraining.

We need telelibrary capability, where we or a clinician can tap in from anyplace in the world to the resources of any medical library or medical library data base, all the way up to, of course, the National Library of Medicine.
In the Navy Medical Department, we have some of the lowest levels of health care providers forward deployed providing health care to our forces independent of having a clinician present there. I refer to the independent duty corpsmen who accompany the Marine Corps and our smaller ships at sea. These are paraprofessionals who are primary care givers for 200 or more men and women.

We want to be sure as we implement telemedicine throughout the Navy that we are not just consulting back to the specialists at the teaching hospital, which we would see as a vertical implementation of telemedicine. We want to have the capability in place to do horizontal consultation to all levels of expertise. That independent duty corpsman on the frigate in the middle of the Indian Ocean, can consult with a PA as opposed to going back to the surgeon or the orthopedic specialist. We want to make sure that any clinician can consult with ultimately any other clinician from anyplace in the world. That full Web Con activity is one of our principals.

We believe that we face unique challenges in the Marines and the Navy vessels that are on station all the time around the world. We have forces forward deployed around the world. In an operational setting, ships are not linked, they are sitting at harbor, they are at sea, and we need to be able to provide health care to them at sea.

Submarines present a relatively unique communications problem when they are submerged, but we do want to extend telemedicine capability ultimately to all of the operating forces within the Navy. We think that this will change the paradigm of health care delivery. We have already seen in some our prototype trials that we wind up extending all of the clinical expertise at a full teaching facility down to the lowest echelon of health care delivery, which improves the quality of care for every Navy and Marine Corps beneficiary in our system both in the operational environment, which is our first priority, and in the peacetime environment, where we can save funds and clinician time by not having to transfer patients from one point to another for consultations.

We also have some difficult communications issues which we think are unique to the service. You would 
think by looking at an aircraft carrier, or frigates, cruisers or any other ships at sea bristling with antennae, that you would be able to add another antenna anyplace on that carrier and transmit an image anywhere. The problem is that those ships are saturated for communication assets, and they are saturated in physical space, which is no small problem. For example, we learned through a prototype from an aircraft carrier that parking the planes has a detrimental effect on line of sight communications with satellites. The Navy tends to think of it as more than an inconvenience when we ask them not to park planes on a certain part of an aircraft carrier. We need to adapt to their requirements.

What we also find is that there is electromagnetic frequency (EMF) saturation on the decks of the ships. You cannot erect a new antenna and expect it to function on its own. There is always a ripple effect of impact on other communication assets, some we do not even foresee.

On another test from an aircraft carrier, we found that our telemedicine unit was functioning just fine until an EGS cruiser, which has some of the strongest radar units in the world, pulled up along side and blew every circuit in that unit, just from irradiating from about a mile away the telemedicine antenna that we were using.

Again, we cannot compromise the defensive posture and the mission of our operating forces by asking the surface ships to turn off their anti-air radar while they are steaming just so we can transmit telemedicine images. If we are going to implement telemedicine across the entire DOD spectrum, the Navy feels that we have the hardest communications piece to solve. If we are going to have a full continuum of telemedicine transmitting images between multiservice facilities, all of the components need to address the unique communication constraints of the Navy. We think that we are the lowest common denominator on the communication chain.

What we have started to do is integrate with existing line communications assets, and what we see on this slide are some of the assets that we have been testing. Using the defense communications satellites has some serious setbacks for us; there are only two of them, line of sight is difficult and they are very slow. 512KBS is not fast data transmission. We found that these satellites were not adequate for the data transmission that we required.

I spoke about super structure saturation and the EMF interference. Challenge Athena is an example of how Navy telemedicine has tried to integrate with our line counterparts. Challenge Athena was actually an intelligence community exercise, using different electronic transmission capabilities from ships at sea. We piggybacked on top of them and got some very dramatic results, which I will address shortly, from the USS George Washington, a nuclear aircraft carrier, during a six month deployment.

We are also working with the technologies that you see here. I would draw your attention to the bottom of the slide, which I know is difficult to see from the back of the room. That is the USS Abraham Lincoln, which is ready to sail within a matter of days. The Lincoln will be sailing with the most complete telemedicine suite ever fielded to a remote unit for the Navy Marine Corps Team. It will have teleradiology capability, video teleconferencing; not only will it be able to communicate with a full teaching hospital ashore from anyplace in the world during its six month deployment throughout the Pacific and other places, but it will also allow escort vessels to communicate to the aircraft carrier using what is called POTS, one of my favorite acronyms, "plain old telephone system", through cellular and HF channels POTS.

Anybody who has been at sea, and I realize that many of you have not, knows the difficulty in transporting a patient from a frigate in 10 or 12 foot seas either by helicopter, which is not a pleasant excursion by itself, or what we call "high-lining" patients between ships, where you literally string a cable between the ships and, using pulleys, pull the patient to the other vessel. Nobody volunteers for that.

Patient movement at sea from the small vessels to the large vessels for consultation is extremely difficult. The health care continuum will extend to the small vessels with the Lincoln battle group, and we think that we are going to see some dramatic increases again in the quality of health care. It will not be necessary to request that the entire task force stop steaming and bring the escort vessels that are screening against submarines and aircraft into the aircraft carrier, and therefore compromise the defensive posture of the task force.

Moving quickly through the goals for Navy telemedicine, the first is maintaining the operational readiness of the force, and that is key to us. We know from a study that we should be able to eliminate approximately $28 \%$ of the patient evacuations both in peacetime and wartime. That means eliminating $28 \%$ of medivac operations. That means $28 \%$ of the time we will not have to fly replacements into the combat zone for wounded Marines. And $28 \%$ of the time we will not have to compromise the battle group by bringing a vessel closer to shore to fly a patient off.

We also want to use the organic communication systems from the ships and the Marine Corps units that we are supporting, and we want to use commercial off-the- 
shelf products. The Navy Telemedicine Initiative wants to have the leading edge, but there is risk involved in emerging technologies. We feel that the risk should be shared equally between the military services and the commercial vendors who are developing the product, as opposed to testing it with the Navy or any other military service for commercial applications elsewhere. It is that type of partnering agreement that we seek with the different vendors who are working with us.

I will draw your attention here to the George Washington. The George Washington, on a six month deployment in the Atlantic, Mediterranean and Indian Oceans, was able to avoid $41 \%$ of the medical evacuations during that six month deployment. We did pretest measures of how many evacuations normally occurred from a carrier battle group so we could evaluate the effectiveness of the system once it was deployed. We think that that is a key part of implementing this technology. We want to be able to gauge effectively the impact of putting this technology out into the different units, both on the quality of care and the treatment patterns.
As for our hospital ships, the US NS Mercy, which is sailing from the West Coast as we speak, will not only have full telemedicine capability, including still image capability, thanks to a partnering with the Army R\&D, the MATMO command at Fort Detrick, but they will also be linking the Composite Health Care System (CHCS) with that telemedicine component back to the units at shore and to the teaching hospital in San Diego demonstrating that capability.

In the interest of time, let me conclude at this point, and stress that the Navy is actively fielding telemedicine at 12 different sites. We are also partnering with the North Carolina ATM telemedicine network, University of Texas, Texas Medical Center EMS network and others. We are working to ensure that we can put integrated telemedicine capability out to as many sites as possible, well demonstrating a cost benefit payback for the sites that we are putting out to, and for that reason we are prioritizing our deployment to the remote sites and the operating forces, then following with the smaller facilities and ultimately the larger shore base facilities for deployment.

I thank you for your attention this morning. 